$\mathcal{G S}_{\text {https://doi.org/10.3765/sp.10.3 }}^{\text {Semantics \& Pragmatics Volume 10, Article 3, } 2017}$

This is an EARLY ACCESS version of

Vasardani, Maria, Lesley Stirling \& Stephan Winter. 2017. The preposition at from a spatial language, cognition, and information systems perspective. Semantics and Pragmatics 10(3). https://doi.org/10.3765/sp.10.3.

This version will be replaced with the final typeset version in due course. Note that page numbers will change, so cite with caution. 
EARLY ACCESS

\title{
The preposition at from a spatial language, cognition, and information systems perspective*
}

\author{
Maria Vasardani \\ The University of Melbourne
}

\author{
Lesley Stirling \\ The University of Melbourne
}

Stephan Winter

The University of Melbourne

\begin{abstract}
While both linguistic semantics and geographic information science have struggled to accurately and usefully define the meanings of natural language prepositions, there has been less dialogue between these disciplines than one might expect. In this paper we set out to describe the meaning of the English preposition at using well-established formal models of qualitative spatial relations within geographic information science (GIScience), ultimately proposing an account for its meaning which is novel for both discipline areas, and we relate this description to previous definitions of at from within linguistic semantics. The investigation in this paper illustrates that at cannot be handled using the mathematical formalizations of crisp spatial relations in GIScience. However, the model proposed in this paper based on contrast sets, Voronoi Diagrams and Galton \& Hood's (2005) Anchoring theory, contributes to a better understanding of the meaning of at. The model also enables improved automatic interpretations of the preposition by partitioning space into contrast sets and representing $a t$ as indicating that a spatial entity is more closely related to a reference object than to any other in the set. We show how the model can be framed within a formal semantics account and how it can in principle be extended to account for spatial uses of other prepositions as well. The paper demonstrates potential areas of fruitful cross-fertilisation between GIScience and linguistic semantics.
\end{abstract}

Keywords: Spatial prepositions, spatial cognition, qualitative spatial relations, geographic information science.

\section{Introduction}

The linguistic semantics of prepositions has been the subject of much discussion but the definitions which have been proposed are not yet sufficient for practical needs, such as those of geographic information science (GIScience), which conceptualizes

* We thank the anonymous reviewers and particularly Louise McNally for their valuable feedback. 
qualitative spatial relations in formal mathematical models for qualitative spatial reasoning. On the other hand, the formal mathematical models developed by GIScience for topological, distance, and direction relationships incorporate semantic definitions which relate only partially to the way that prepositions are used in natural language utterances (D. Mark \& M. Egenhofer 1995, D. Mark, Comas, et al. 1995, Worboys 2001). In this paper we seek to bring the work done in these two discipline areas closer together. We take as our case study the qualitative spatial relationship that is expressed by the preposition at, which is not adequately covered by any of these models, raising the question as to why such a prominent preposition has been ignored so far.

$A t$ is an interesting preposition in that it is highly underspecified, and can correspond to multiple, often more precise types of spatial situation in context. For example, let's meet at the park may imply a meeting point inside the park, a meeting point along its boundaries, or even a point outside the park, but close to it. The interpretation of the preposition depends here solely on the communication partners' implicit understanding. If somebody types into a search engine 'café at the park' the relevance or ranking of individual cafés would depend on the unspecified context: is the person interested mostly in cafés in the grounds of the park, attached to the park, or in reasonable proximity to the park? Or is an implicit ranking more appropriate, for example, such that cafés on the grounds of the park are more relevant than cafés within a reasonable distance from it?

Hall, Jones \& Smart (2015) show that at is the most frequently used spatial preposition in the database of geo-referenced photo captions they analyse. This makes a formal mechanism for processing and generating at particularly desirable: if natural language processing can be improved by capturing the meaning of at by formal models, place descriptions containing at can be interpreted automatically, or in the case of the work being done by Hall, Jones \& Smart, photo captions can be generated automatically. As a result, human-machine interaction involving natural language would be improved. Current search engines still ignore spatial prepositions such as at in queries as being too hard to resolve (see example query above). A formal model should also be consistent with cognitive models of the meaning of at, and allow qualitative spatial reasoning over it. In fact, formal models capturing the meaning of at may also contribute to our understanding of cognitive processes and their reflection in qualitative spatial reasoning.

This paper starts from the hypothesis that the meaning of at can be formally captured by tools of GIScience. The method applied looks at the discriminatory power of at in comparison to the known qualitative spatial relationships or operators, described in more detail in Section 3. It will reveal that at has no topological discriminatory power, only a ternary distance discriminatory power, and no directional discriminatory power. At least the first two observations may be surprising, given 
the usual specializations of $a t$ in GIScience by the set of two topological descriptors (coincidence, adjacency) and a binary distance descriptor (proximity), and the approaches to the description of its meaning current in cognitive and formal semantics. The paper then explores whether the meaning of at can be captured by a combination of a contrast set approach (S. Winter \& Freksa 2012), the use of Voronoi Diagrams, and the theory of Anchoring (Galton \& Hood 2005). We conclude that such an account does provide the tools to capture the lexical meaning of $a t$.

In Section 2, we briefly survey relevant previous research on the semantics of at. In Section 3 we work through and evaluate a range of formal models for the spatial meaning of at from GIScience, concluding that a novel model is needed, based on the combination of contrast sets, Voronoi Diagrams and the notion of Anchoring; this is described in Section 4. In Section 5 we relate this account to existing theories within linguistic semantics, and in particular show that it can be incorporated into the kind of compositional model-theoretic formal semantics assumed by Zwarts \& Y. Winter (2000). Finally, in Section 6 we look at some complex cases and the issue of over-generation of the account, identifying some questions for ongoing research and arguing that a formal account such as the one we propose requires supplementation with a neo-Gricean pragmatic approach. Although our primary focus is on at for the reasons given above, along the way we also illustrate how the proposed account can apply to and distinguish at from other English prepositions, in particular in, on and near.

\section{Related work}

The preposition at has a range of meanings or use types, including at least those listed in Table 1.

Like other prepositions, at is thus clearly polysemous; however, we focus on the spatial meanings here (cf. also Herskovits 1986 and others), aiming to give a general unified representation applicable to these uses. We make the assumption that the temporal and metaphorical extensions can be handled by a relatively straightforward mapping of the spatial meaning onto a different domain. The mapping can be done while maintaining the same general properties and with additional conventionalised elements of meaning resulting from contextually common inferences. ${ }^{1}$

1 For example, temporal uses appear similar in structure to spatial uses if we assume an ontology of points in time and that intervals of time (e.g.. seasons) can be viewed punctually. Crucially, in See you at $4 o^{\prime}$ clock it is understood that I am not behaving uncooperatively if I arrive at a few minutes before or after 4 . To anticipate our account, the reference time belongs to a relevant contrast set where segments are measured probably in 5 minute units rather than more precisely, while in other contexts a more precise specification may be relevant. 
- Spatial locational

a. I will meet you at Melbourne Central.

b. John is at the tram stop.

c. The butterflies live at the park. ${ }^{2}$

d. Put the posters up at the Engineering buildings. ${ }^{3}$

e. We arrive at Heathrow in 30 minutes.

- Functional

f. Are you at your desk?

g. My daughter was at the piano when the doorbell rang.

h. I'll be at work from 8 am.

- Temporal

i. See you at 4 o'clock.

j. There aren't many birds at this season.

$\mathrm{k}$. The exam begins at precisely $2.00 \mathrm{pm}$.

- Other metaphorical extensions / idioms

1. At the height of his success, he was struck down with illness.

m. The children are at play.

n. The chickens have been at the corn again.

o. If you look carefully at the painting you can see a reflection in the mirror.

p. She yelled at me when I tried to get her to move.

q. He was really upset at the news of this company's closure.

r. John threw the ball at me.

s. We pushed at the door but it would not open.

Table 1 Major uses of at. 
The preposition $a t$

Lexical items, if meaningful, should carry some discriminatory power. That is, they should have information content in the sense of distinguishing between possible states of the world, and convey the chosen state to a recipient who does not know. This latter aspect of novelty or surprise for the recipient was behind the original definition of information content from information theory, which had a need to distinguish between information and noise in a signal (Shannon 1948, Shannon \& Weaver 1949).

Closed class words, such as conjunctions and prepositions, have a different effect on human cognition than open class words such as nouns and verbs (Bloom \& Keil 2001). Usually the meanings of prepositions do not overlap, thus there is little choice amongst lexical terms for describing the spatial layout of objects (Bryant 1997, Landau \& Jackendoff 1993). Some prepositions, however, are less exact, and at is one of them. The precise spatial array referred to with a use of at is determinable based on the context of use rather than residing in the semantics of at itself (Bennett 1975, Herskovits 1985, 1986). The prevalent assumption in linguistics states that $a t$ may be used in place of more specific spatial expressions such as on, in, touching, and near (Cienki 1989, Cuyckens 1984, Herskovits 1986), suggesting a relationship of hyponymy - a hierarchical or inheritance relationship — between these more specific spatial relationships and at, rather than the relationship of mutual opposition assumed in the above characterisation of prepositional meaning. We have previously suggested that preferred meanings of one of these more specific prepositions are prevalent in place descriptions with at, depending on the type and spatial granularity of the referents (Vasardani et al. 2012).

Furthermore, it has been shown that there is a good deal of cross-linguistic variation in prepositional meaning (cf. for example Levinson 2003), and it is also worth noting that the preposition at is not universally matched with translation equivalents across languages. For example, Polish has multiple prepositions capturing aspects of the meaning of at (Knas 2006), and in German one would use in some cases an, in others zu or bei. Hence, Cuyckens (1984) calls at a typically English preposition. Other authors have pointed out similar differences between languages for other prepositions, supporting more general views that "there must be universal non-linguistic foundations for spatial language, and that there must be language-specific effects on spatial representations" (Munnich, Landau \& Dosher 2001: 172-3).

Much previous work on the semantics of at has attempted to capture our intuitions about the meaning of at within a cognitive semantics framework. Like other accounts within linguistic semantics, this work assumes that in their core meaning prepositions code spatial relationships between two entities. We will refer to these

2 Example as discussed in Galton \& Hood (2005).

3 Example from the corpus analyzed in Vasardani et al. (2012). 
here as the locatum (the located object, identified somewhere in the discourse external to the prepositional phrase), and a reference object, with respect to which a region is identified providing the location of the locatum (usually specified in the noun phrase complement of the preposition). In cognitive semantics, the locatum is called a 'trajector', and this trajector is located with respect to a 'landmark'. Other terms which have been used for this contrast are 'figure' and 'ground'. Landau \& Jackendoff (1993) discuss some differences between the concepts 'reference object', 'landmark', and 'ground'.

One key insight from the work in linguistics has been that the preposition at expresses a topological relation of co-location - coincidence or adjacency — between these two entities, with the location conceptualised as a point and without any specification of a direction of orientation of the locatum (Evans 2010, Herskovits 1985, Leech 1970, Tyler \& Evans 2003). Evans (2010) specifies a co-location lexical concept licensing spatial uses of at and encoding the co-location parameter, which designates an abstract spatial relation between the trajector and a location defined in terms of contiguity or proximity (this alternative of coincidence or proximity dates back to at least Cuyckens 1984). The explicit acknowledgment of the irrelevance of the landmark's dimension or the perception of it as a point also dates back to early work (Leech 1970, Lindstromberg 1998, Quirk et al. 1985, Tyler \& Evans 2003).

It is this zero-dimensional (point) status of the location which has been thought to underlie the observation that a spatial situation described by at can have a range of specific instantiations: if the park in the 'café at park' query example given above is treated by at as zero-dimensional, then the description simply says nothing further about where the locatum is with respect to this point, the key complication being that in 'real life' of course the park is not zero-dimensional and hence the true relationship between the locatum and the park must be more specific. Thus it is acknowledged that $a t$ "affords the most general expression of localisation in space in English" (Evans 2010: 243). On the same page Evans also notes that all the more precise expressions of spatial relationships encoded by English prepositions such as by, near, on, in, over, under "at times, can be encoded by at". The base generality of at is confirmed by its ability to non-redundantly co-occur with specifiers such as precisely or exactly.

In addition to work in cognitive semantics, formal semantic models of prepositional meaning have also been proposed, framed within compositional modeltheoretic approaches (Gärdenfors 2014, Zwarts \& Y. Winter 2000). These approaches generally account for the spatial meanings of locational prepositions by treating the preposition as a function over the spatial realisation of the reference object (encoded in the preposition's noun phrase complement), which defines a region within which the locatum is to be found: the preposition then contributes information which allows us to determine how the region (the location of the locatum) is related to the location 
of the reference object. Within such approaches, a number of claims have been made about the semantics of at. For example, Zwarts \& Y. Winter (2000) propose a model in which spatial meaning of prepositions is accounted for using vector spaces. They make several major claims about the meaning of at, such as that it belongs to a class of prepositions giving rise to regions outside the eigenplace ${ }^{4}$ of the reference object and, similarly to the observation about contiguity above, that at imposes the sole additional constraint that the length of the vector (distance between locatum and reference object) is "almost zero". However, their account says nothing further about the meaning of at and does not, for instance, distinguish it formally from on, so that for example the formal semantics of they are at the supermarket is not distinguished from that of they are on the supermarket (that is, physically on the roof of the supermarket) (for more information see Section 5).

A second feature of the meaning of at identified in the literature has been that at least a subset of uses of at imply a functional relationship which Evans (2010: 243) calls 'practical association' (see also Herskovits 1986): thus, if I am at my desk the implication is that I am working there; if I am at the bus stop that I am waiting for a bus, and if I am at work or school or university that I am there to fulfil the normal functions I would undertake in that place, or even that I work or study there, regardless of where I am actually physically located at the time of utterance (see Table 1f-h above). Thus for these uses the type of activity which would normally be undertaken at the locatum is highly relevant. While it is important to account for these interesting uses within a full description of prepositional meaning and usage, it is not clear to us that this should be incorporated within the lexical meaning of the preposition rather than as a generalized conversational implicature. Intention or function remains context delimited, it is just that some contexts are so common as to become typical (when at the piano I would normally be seated facing it in a position to play, but in context I might be understood to have a different orientation to it, for instance if I am about to make a toast or give a speech). Similar pragmatic implications can be found with at least some other prepositions: if I say I am in bed, you are likely to understand me to have retired for sleep for the night or to be unwell, depending on context; if I say I am in the car you will likely understand me to be driving from place to place, while if I am on stage I am likely to be performing in a production - however, all these implicatures can be cancelled. We therefore do not tackle these uses here.

4 The eigenplace is the spatial region occupied by the object (Wunderlich 1991). 


\section{Evaluating formal GIScience approaches to defining the meaning of at}

Over the past decades GIScience, in collaboration with artificial intelligence, has developed a rich body of knowledge about conceptual representations and reasoning with qualitative ${ }^{5}$ spatial relations (Cohn \& Renz 2008, M. J. Egenhofer, A. U. Frank \& Jackson 1989, A. U. Frank 1991, Freksa 1991, Kuipers 1994, 1977). The qualitative turn in the discipline was motivated by the recognition that qualitative reasoning is computationally faster, due to its higher level of abstraction, than quantitative evaluations, and in many tasks sufficient. But from the beginning, also, the adequacy of these models for cognition and language was considered (M. J. Egenhofer \& D. M. Mark 1995, A. U. Frank \& D. M. Mark 1991, D. M. Mark \& M. J. Egenhofer 1994, D. Mark, Comas, et al. 1995, D. Mark, A. U. Frank, et al. 1989, D. M. Mark \& A. U. Frank 1991).

In this section we work through key formal models from GIScience of the qualitative spatial relations of topology, distance, and direction, considering the meaning of at within each of these. Although there is a limited amount of work on at in GIScience, these form the current 'toolkit' available to GIScientists to formally define the meaning of $a t$ and other prepositions, and some proposals have been made for at within them (cf. Dube \& M. J. Egenhofer 2012).

\subsection{Topology}

GIScience has developed two formal models of topological relations, the 9-intersection model based on point-set topology (M. J. Egenhofer, Sharma \& D. M. Mark 1993, M. J. Egenhofer \& Franzosa 1991), and the region connection calculus based on first order logic (Cui, Cohn \& Randell 1993, Randell, Cui \& Cohn 1992). Both models, despite their different foundations, result in definition of the same eight basic topological relations between two simple regions (abstractions of geographic features). Let us pick the 9-intersection model for illustration (Figure 1). In the 9-intersection model these eight relations are called 'disjoint', 'meet', 'overlap', 'equal', 'coveredBy' and its inverse 'covers', and 'containedBy' and its inverse 'contains'. They are characterized uniquely by the observation as to whether the intersection of the interiors, boundaries and exteriors of the two regions are non-empty or empty. For example, the relation 'overlap' is the only relation where the two

5 Qualitative spatial relations are symbolic abstractions of geometric representations of features, while quantitative relations express metric values. Qualitative relations allow spatial analysis, which is independent of, but consistent with the geometric depictions (M. J. Egenhofer 2015). $X$ is 100m away from $Y$ is an example of a quantitative relation, while $X$ is far from $Y$ is an example of a qualitative relation. 


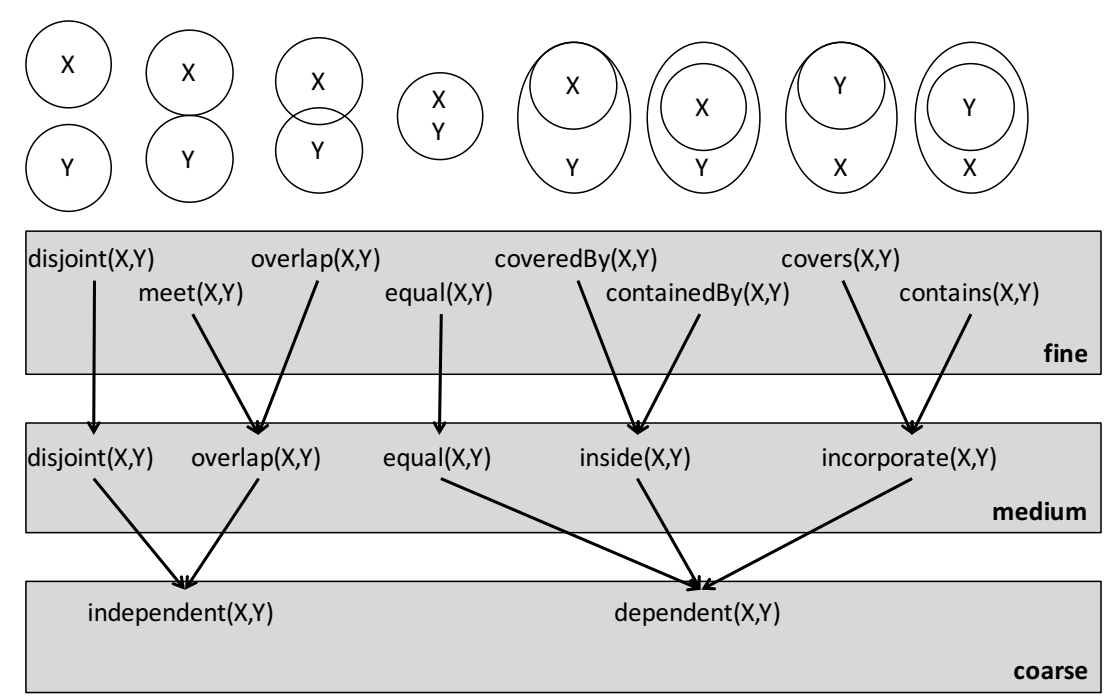

Figure 1 The eight topological relations between simple regions in the 9intersection model, and their generalizations.

interiors intersect, the two boundaries intersect, and parts of each region's interior are outside of the other region.

For geographic databases, storing these relationships in addition to the actual geometry of geographic features speeds up search. For example, finding all regions 'meeting' a given specified other region, or all regions 'overlapping' with a specified region, no longer requires costly geometric operations. In the context of the current paper these eight basic topological relations are relevant as possible tools that can be used in order to characterize the meaning of at.

When we evaluate the meanings of at with regard to these conceptual models, we find that the various uses of at can cover all eight possible topological relationships between two regions, or that at has no topological discriminatory power, in contrast to, for example, prepositions in, on, and near, each of which corresponding to a single or a small subset of possible topological relations. Dube \& M. J. Egenhofer (2012) claim that at can be modelled as the union of 'equal', 'coveredBy' and 'containedBy', but do not discuss natural language examples.

- If reference object and locatum are disjoint, at is applicable in cases of proximity: If $A$ is near $B$ one may say $A$ is at $B$, especially if thinking of or looking at $A$ and $B$ from a distance, or where $B$ is a local or global landmark recognizable by many, in contrast to a relatively unknown $A$. 
- If reference object and locatum are in contact at their boundaries, this contact can be external contact ('meet') or internal contact ('overlap', and potentially cases of 'coveredBy', 'covers', or 'equal').

- Two adjacent buildings that share a wall: The new maternity wing is at the east side of the hospital ('meet').

- A complex construction may contain sub-structures visible at street level: I mean the cafeteria at the ground floor of the hospital ('coveredBy').

- Two buildings extending over a common area: The car park is at the ground floor, leading to the mall from its north exit and to the train station from its south exit ('overlap').

- Finally, the sentence: A new hospital wing is being built at the parking lot ('equal') is also a valid use of $a t$.

- Example sentences for 'coveredBy' and 'covers' relations, requiring a contact at the boundaries, seem to be indistinguishable from sentences that exemplify 'containedBy' and 'contains'. Hence, 'on' (surface) or 'in' (container) uses of $a t$, relating indistinguishably to 'containedBy' and 'contains' as well as to 'coveredBy' and 'covers' relationships, occur in topological examples as well:

- The new central station was built at the place of first settlement may be an example of 'contains' (or 'covers'), assuming the areal extent of the central station is significantly larger than the three huts forming the first settlement.

- I am at home, answering a phone call while sitting in the living room, is an example of 'containedBy' (less likely, 'coveredBy').

This observation is even more relevant as the eight topological categories formed by the 9-intersection model (and equivalently by the region connection calculus) have been shown to be the most promising starting point for further investigating how people conceptualize topological knowledge: although grounded in point-set topology (or first-order logic, respectively), their distinctions have been shown to reflect configural situations which humans would cluster together, or situations which language has names for (Knauff, Rauh \& Renz 1997, Renz, Rauh \& Knauff 2000).

Both models, 9-intersection and region connection calculus, have been abstracted to five more general relations, but at this (medium) level of abstraction, 9-intersection and region connection calculus show differences in the way they group the relations. The medium-level 9-intersection model (Figure 1) emerges if boundaries of regions 
are considered to be parts of regions, therefore when the boundaries externally meet or intersect, they are grouped together under (medium-level) overlap. However, since at did not discriminate between the original eight relationships, it also does not discriminate between any of these five medium-level relationships, regardless of the model.

Figure 1 shows a further level of abstraction, grouping the five medium-level 9-intersection relations into two clusters only. One cluster aggregates all relations where portions of the boundaries of both regions are outside of the other region, while the other aggregates all relations where the boundary of one region is completely within the other region (remembering that the medium-resolution 9-intersection considers boundaries as part of regions). The same clustering works for the region connection calculus.

One can further argue that these two most general clusters, 'independent' and 'dependent', must represent the coarsest topological distinction, and therefore, a cognitively essential distinction. And yet, cases of applications of at still can be found at both sides of the divide. For example, a meeting at a café that is a separate building, but very close or even attached to the National Museum may be expressed as in Let's meet at the café at the National Museum; this represents the 'independent' case. Alternatively, if two friends want to look for gifts, they can meet at the Museum's gift-store - which is part of the museum, so that the relation between museum and gift-store falls under the second general cluster of the 'dependent' relation - as in There is a gift-store at the museum, let's go there. Hence, it appears that at has no topological discriminatory power, and is vague in terms of topological meaning, at least assuming it is desirable to practice parsimony with respect to multiple polysemous senses. This conclusion is in accordance with the prevalent assumption in the literature of the irrelevance of the dimension, or the zero-dimensionality, of the perception of the reference object in most spatial uses of at. We will refer again here to the let's meet at the park example. Since at has no topological discriminatory power, it really does not matter whether the sentence is interpreted as meeting inside the park, or just outside of it, and therefore, the park's conceptualization as either point or extended feature is unimportant. What really matters is the mutual knowledge between the conversation participants as to where to meet. In contrast, when in or on are used the higher-than-zero dimensionality of the landmark is important for a topological interpretation. In the same example, let's meet in the park, means that the park is conceptualized as a two- or even three-dimensional feature and the people will meet inside of it. 
Vasardani, Stirling and Winter

\subsection{Distance}

The most prevalent meaning of at is a distance-related one, and this is reflected in the core linguistic semantic definitions of co-location-contiguity / proximity, as well as the 'near zero length' vector in the formal semantic approach of Zwarts \& Y. Winter (2000). However, at has not been discussed (perhaps has not been perceived) in the GIScience literature as a qualitative distance relationship (Hernández, Clementini $\&$ Di Felice 1995). The qualitative distance relationship closest to the meaning of at is 'nearness' (Brennan \& Martin 2002, Worboys 2001). But even if nearness is seen as an abstraction from quantity of distance in such accounts, clearly we still need to distinguish the meaning of at from the meaning of near, because unlike at, near cannot represent the full range of topologically dependent relationships demonstrated above (it cannot include spatial arrays of meeting or containing), because the distance-related dimension of meaning encoded in near is greater than the 'near zero length' vector at is assigned in formal semantics. For example, it is valid to say the café is at/near the museum if the café is very close to the museum. However, if the museum includes a café, it is valid to say the café is at the museum, referring to the collocation of the café with the museum's grounds. But it is not valid in this case to say the café is near the museum.

We suggest here that the meaning of at can be more appropriately formalized by starting from the concept of a contrast set of locations (S. Winter \& Freksa 2012). With such a set of contrasting locations in mind, the meaning of 'at a location $A$ ' is limited to locations which are closer to $A$ than to $B$ or to any other alternative location to $A$ in the given contrast set - assuming that the contrast set forms part of the context of the conversation. Taking up an argument by Brennan \& Martin (2002), at some level at therefore reflects not a binary absolute proximity relation (cf. 'near $A$ '), but a ternary comparative proximity relation: ' $X$ is nearer to $A$ than any other alternative(s) of the contrast set' ${ }^{6}$

S. Winter \& Freksa (2012) argue that places - the elements in these contrast sets - are not characterized by boundaries. Places are rather characterized by prototypes or centres, or are even conceived of as dimensionless entities in information space. The spatial extent of a place is only a refinement, not the place's nature. In addition, the spatial extent is frequently vague or contested (Burrough \& A. Frank 1996, Cresswell 2004). Thus, this approach of reducing places to points fits well to the observed lack of topological discrimination of at but also to dealing with proximity: distance between, or proximity to, dimensionless entities is cognitively and computationally more directly accessible than distance between a point and

6 Note that something can be both near $A$ and near $B$ even if $A$ and $B$ are not co-located - but a locatum cannot be both at $A$ and at $B$ if there is not a close spatial relationship between $A$ and $B$; see the formal treatment proposed in Section 5. 
a spatially extended region (again, abstraction of a geographic feature or place), or between two spatially extended regions. Such distances would require first a commitment to the region's spatial extent, and then more complex calculations such as that of distance between a point $x$ and an extended place $A$, as in this definition of Mador-Haim \& Y. Winter (2015: 448), where $y$ is a point in place $A$ (Eq. 1):

$$
d(x, A)=\min (\{d(x, y): y \in A\})
$$

or for two extended places the Hausdorff distance between extended place $A$ and extended place $B$ (Hausdorff 1914). The Hausdorff distance is the greatest of the distances from any point $a$ in $A$ to the closest point $b$ in $B$ (Eq. 2). The 'suprema' (sup) and 'infima' (inf) are used for calculating the longest distances (suprema) among the closest (infima) of the points between the two places:

$$
d_{H}(A, B)=\max \left\{\sup _{a \in A} \inf _{b \in B} d(a, b), \sup _{b \in B} \inf _{a \in A} d(a, b)\right\}
$$

The geometric interpretation of the comparative proximity relationship argued for by S. Winter \& Freksa (2012) is given by a Voronoi Diagram (Voronoi 1908). A Voronoi Diagram partitions space between seeds such that each point in space closer to one seed than to any other belongs to the cell of that seed. Voronoi diagrams always produce convex cells, and thus this formal model is aligned with Gärdenfors' thesis that "prepositions represent convex sets of points or paths in a single domain" (Gärdenfors 2014: 205), except that it identifies this set of points as a Voronoi cell. It is also in some sense aligned with the 'near zero length' vectors of Zwarts \& Y. Winter (2000: 176-7), but with the assumption that specifying the maximum length of these vectors as is done in the Voronoi cell model is essential for their interpretation. And the discussion so far contradicts the assumption of these authors that prepositions are either outward or inward oriented: at appears to be valid outwards (near) as well as inwards (in, on). This will be explained in more detail in Section 4.

On this account the seeds of the Voronoi Diagram are the places $A, B$, and so on, of the current contrast set. For example, Figure 2 shows the Voronoi Diagram of a contrast set of inner city train stations in Melbourne, Australia. According to what has been said above about the distance meaning of at, in a communication context where this is the relevant contrast set - say, a person on a train calling home - the sentence: I am at Parliament Station is acceptable if used when the speaker is inside of the station as well as when they are on a train approaching this station or departing from this station as long as the train is nearer to Parliament than to the next (Melbourne Central) or previous station (Flinders Street). This example is purely locative, not directional. Cases of direction or orientation are discussed in Section 3.3. 


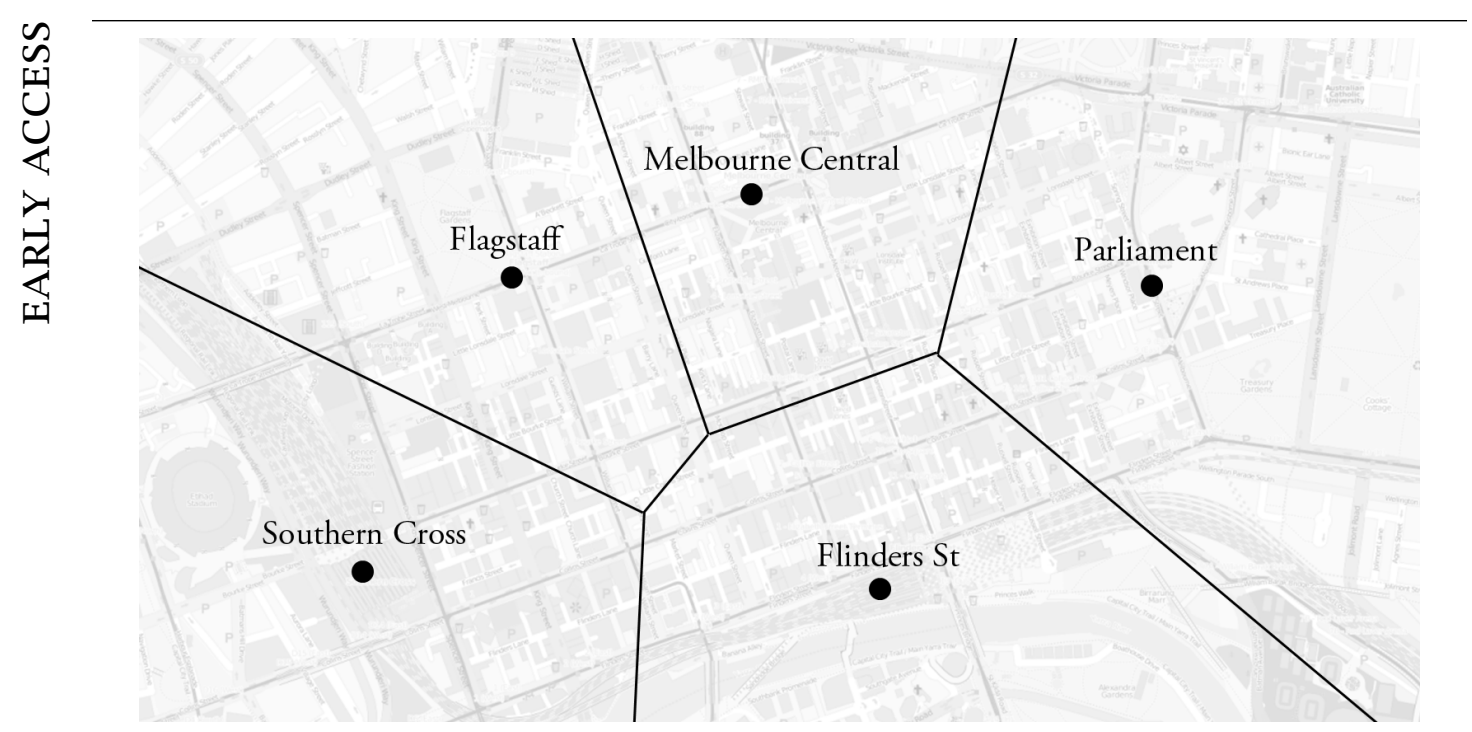

Figure 2 The Voronoi Diagram of the set of Melbourne's inner city train stations.

It should also be noted that this account aims to represent space partitioning at a cognitive rather than at a physical level. What this means is that at will not be equally used when referring to places closer to the borders of the Voronoi cells as when indicating places closer to the centre. One way to think of this is to see the influence regions surrounding the seeds of the cells as operating like gravitational fields, with forces stronger closer to the centre of the field and decreasing in intensity the farther away one moves from the centre. This in turn affects the likely use of the preposition at according to how close or far one perceives they are from one centre in the influence region. This implies that while we are using the geometry of the Voronoi diagram to delineate the influence regions of a contrast set, we are not claiming that the use of at can partition the physical space fully. Other prepositions such as between may apply better, if the location referred to is closer to the border of the Voronoi cells, and neo-Gricean principles of pragmatics will ensure that in such a context speakers will choose the more specific representation of the spatial relationship, if they are aware of it. ${ }^{7}$

Brennan \& Martin (2002) in their work on nearness, propose the use of Power Diagrams, or weighted Voronoi Diagrams. In Power Diagrams the seeds have various weights, for example, according to their prominence or relevance. However, for

7 As Gärdenfors (2014: 26) points out in his model of conceptual spaces underpinning his theory of linguistic meaning, which makes also use of Voronoi Diagrams, the convex influence regions of the Voronoi cells are not homogeneous, which is what makes them suitable for representing prototype effects; the seed of the cell can be seen as the prototype case for the concept and different positions within the region are describable as more or less central depending on their distance from the seed. 
contrast sets this suggestion seems to be inappropriate: a contrast set collects equals as alternatives, features of the same kind, of similar prominence, but most importantly of similar relevance in the given context. If the purpose of a sentence is to position a locatum, the reference object will be chosen from a set of similarly relevant alternatives. If the concert will be played at the city hall, this means just that it will not be played at the other possible well-known event locations in the city. This contrast set does not contain locations that are irrelevant in this context, such as, normally, my office, or Fiji.

Contrast sets are chosen pragmatically, and the semantics of the preposition merely tells us that such a set must be appealed to, not what the set is in a particular context. The set chosen reflects the most relevant set of geographic features or placenames among which the referent object can be situated. While this focus is concerned only with selecting features (encoded in noun phrases in language), there are consequences for the interpretation of at, including its validity in a particular communicative context. Imagine the following dialogue as an example (Table 2), where the context, and hence, the contrast set changes with nearly every line (here ' $<>$ ' is used to denote 'not at', and the features listed in the right column represent the possible alternatives in the relevant contrast sets):
A I am now at Melbourne Central.
(<> Parliament, Flagstaff)
B At the Elizabeth St exit?
( $<>$ Swanston St exit, platform)
A No, at the Swanston St exit
(<> Elizabeth St exit, platform)
B Ah, I see: You are at the State Library. (<> mall, Lonsdale Street)

Table 2 Dialogue with changing context.

Clark \& Wilkes-Gibbs (1986) have shown the collaborative effort that goes into such a conversation. The first expression may have been used to contrast with the other train stations in Melbourne, as in the example above. The second and third, however, zoom in to a finer level of granularity. They switch to a local contrast set of, say, central elements of the train stations such as their platforms and exits. For the interpretation of at in lines 1-3, it is essential to recognize that the spatial expression in 1 and those in 2-3 belong to different contrast sets. This is signalled in part by use of the definite article in 2 and 3, and in part by other processes of inference in the context of the question-answer sequence in progress. The preposition at in the first expression would be interpreted using the Voronoi cell of 'Melbourne Central' in Figure 2. For the communication partner this was not sufficiently detailed, so the clarifying question At the Elizabeth St exit? switches to the proximity relations of another contrast set represented in the Voronoi diagram of Figure 3. Expressions 2 and 3 do not construct a contrast between the exits and Melbourne Central, but only 


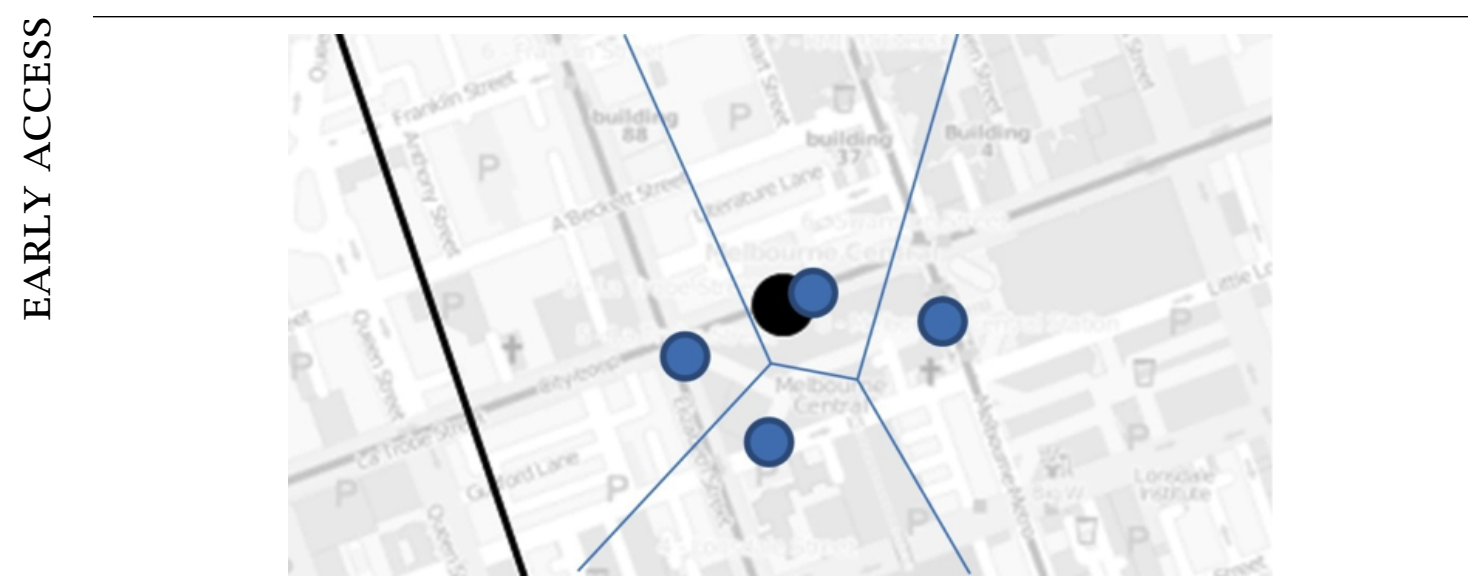

Figure 3 Switch from the original contrast set seed, the Melbourne Central train station, to its possible exits.

among the possible exits (and perhaps similar salient features) within the Melbourne Central train station.

The fourth expression, however, is different. It refers to a feature of similar spatial granularity and prominence as Melbourne Central — the State Library — which is at the Swanston St. exit of Melbourne Central: across the street, but actually closer now than the prototype concept of the train station, with its tracks and platforms. A person leaving Melbourne Central through the Swanston St exit, may, due to the relative low prominence of this exit in the local environment, refer to more prominent features in the environment, again switching contrast sets: away from exits, and picking instead local landmarks. Hence, the contrast is here made with Melbourne Central, but the context has switched from a train trip to everything in local walking distance. Accordingly, the contrast set has changed to Figure 4.

Thus, over these few lines the way in which each prepositional phrase is interpreted in order to establish a location has changed because the context, represented by the contrast set, has changed. The interpretation of at has not changed; in all cases the Voronoi Diagram has catered to a ternary comparative distance interpretation (exit closer to State Library than anywhere else in the contrast set).

Importantly, and similar to the models of topological relations discussed earlier, the lack of discriminatory power of at when it comes to distances relates to the fact that when the actual distance between the locatum and the reference object is important, other prepositions such as near, next to or close by are preferred. In case, however, the notion of general proximity to the specific landmark in implicit contrast to the other landmarks of similar salience is important, then at is the preferred preposition. 


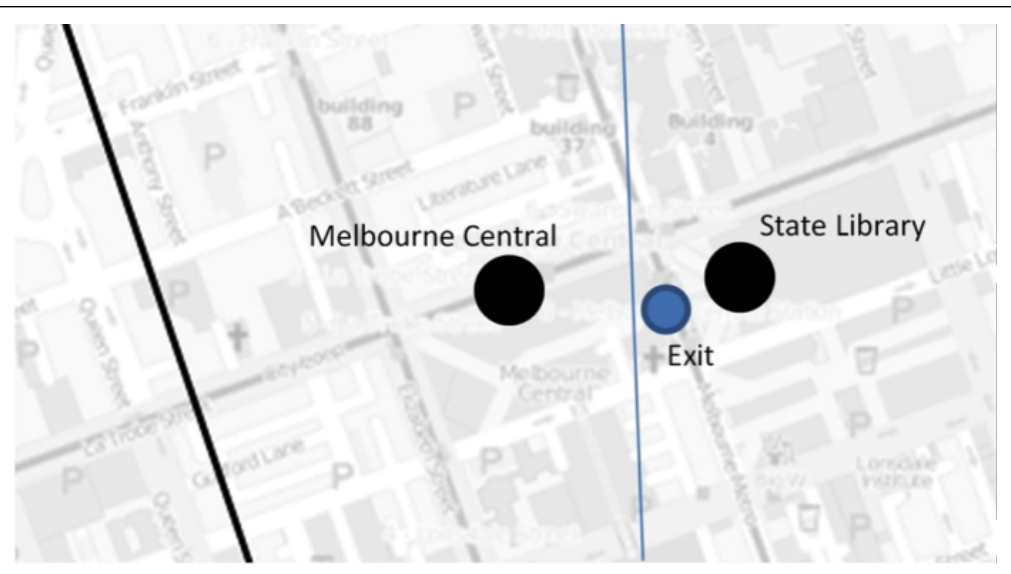

Figure 4 Switching to local landmarks, and now the exit is in proximity to the State Library.

\subsection{Orientation}

When mapping linguistic spatial terms onto spatial relations, there exists a starting point with respect to which the rest of the object(s) are spatially defined. This point corresponds to the origin of a reference frame, at the intersection of three orthogonal axes (Carlson-Radvansky \& Irwin 1994), whether the reference frame is absolute, intrinsic or relative (Levinson 1996). For example, in the sentence the gym is to the north of the student union, the student union forms the origin of the cardinal direction reference frame, to the north of which - along the axis that points North - is the gym. With respect to orientation, at does not relate to any frame of reference. Thus, at cannot differentiate within an absolute reference frame, say, of cardinal directions (A. U. Frank 1996), nor can it discriminate within an intrinsic (object-specific) or a relative (projected) reference frame, for example, left-right or front-back (Freksa 1992).

However, at can also be used with respect to the qualitative relationship 'towards', linked to directed action. In a journey scenario, at can be used to identify waypoints along the route, or also the endpoint. For example, if a plane is arriving at Heathrow, a direction of movement (from an unspecified place) towards Heathrow Airport is expressed, reflecting the assignment of the semantic role of a goal, or endpoint along a path invoked by the motion verb arrive, to Heathrow. ${ }^{8}$

8 While in such uses at can represent the destination location for a verb implying a trajectory along a bounded path, it is distinct from clearly directional prepositions such as to in that while to represents the path as well as highlighting its endpoint, at just represents the endpoint; at also co-occurs with a more limited range of motion verbs than to. For instance, at does not co-occur with verbs of locomotion with a directional sense - Mary walked at the library means only that she walked around 
Thus, while at does not discriminate between absolute, intrinsic or relative orientation, expressions containing at can refer to the orientation of the vector, as in 'arriving at'. Here the motion aspect comes from the verb, while at is used to draw attention to the end of the vector, the target place. Then consistency with the other kinds of relationships requires that source and target are 'disjoint' (as in Figure 1), and some notion of proximity must apply to allow the interaction, although a weak one. For the plane interacting with Heathrow it is likely that the plane is near Heathrow, but it is not essential that it is nearer to Heathrow than to another airport in the region (especially considering the high density of international airports around London in this example). Our intuitions are that the contrast set here is likely between source and goal, so, formally, if the plane is in the second half of its journey it is 'approaching Heathrow'. Similarly, on a train ride from Melbourne Central (start) to Flagstaff (goal) the train can be considered to be 'arriving at Flagstaff' after travelling at least half of the route between these two consecutive stations. ${ }^{9}$ On a car ride from Melbourne to Adelaide the car can be 'at the Grampians', even if it is not a stop, if it is in the Voronoi cell of the Grampians among a relevant contrast set (of well-known places between Melbourne and Adelaide) (see formalization in Section 4). The ternary proximity relation that was postulated above to express the essence of at is preserved. Hence, at combined with a directed action verb or noun is compliant with the previous interpretations. The ternary proximity relationship signalled by the preposition is simply applied to the terminal of the interaction vector.

\section{Formalizing at with Anchoring relations}

As mentioned earlier, the geometric partition of space suitable for the comparative proximity relationship assumed by at can be formed with a Voronoi Diagram, the seeds of which are the places that belong to the pragmatically determined contrast set. Each Voronoi cell includes all the points in space at which a locatum $X$ can be placed in relation to a reference object $Y$, when interpreting the expression $X$ is at $Y$, and

in the influence region defined by the library, and *I am heading at the library is not acceptable. It is presumably this use of at that underlies the metaphorical extension in expressions of looking (look at) and communication (yell at) as well as the sense which contrasts throw at with throw to (see Table 1). See (Jackendoff 1983, Piñón 1993, Snider 2010, Zwarts 2005) for more discussion of paths, directional spatial prepositions and relevant senses.

9 As pointed out by a reviewer and the editor, intuitively there are differences in interpretation between alternative expressions such as $X$ is at Heathrow / arriving at Heathrow / approaching Heathrow / en route to Heathrow (and similarly for any other upcoming stop on a journey, such as Flagstaff station in the example given) - primarily in terms of the likely distance from the target at time of utterance. We make the same point here as in Section 3.2, about the greater likelihood of use of at closer to the seeds of the Voronoi cells. The difference between at Heathrow and arriving at Heathrow presumably reflects the meaning contributed by the use of the motion verb, which entails a trajectory ending in the goal. 
The preposition $a t$

forms the influence region of $Y$, against the rest of the reference objects in the same contrast set. The dimension of $Y$ is not important - as far as its conceptualization is concerned, $Y$ can be thought of either as a point or a 1-, 2- or 3-dimensional object that occupies an eigenplace (Wunderlich 1991), consistent with the actual location of $X ; X$ can in fact be located inside the reference object or within its influence region.

With such a space partition, the formalization of at as a comparative proximity relation can be suitably defined using the Anchoring theory developed by Galton \& Hood (2005). The main premise of Anchoring is that an object in the information space of the unfolding discourse can be anchored to a location in precise (geometric, coordinate-based) space of the world referred to just by stating what is known with certainty in this information space and leaving the rest for further reasoning, thus allowing for the description of qualitative spatial relations which are less than completely explicit. An object $A$, of which the location is known only vaguely or with uncertainty, may be anchored to a location in precise space, a region $R$, by at least the following relations:

Definition 1 The anchoring relations:

- $A$ is anchored in $R$-we know for sure that $A$ is located within $R$

- $A$ is anchored over $R$-we know for sure that $R$ falls within the location of $A$

- A is anchored alongside $R$-we know for sure that $A$ is located such that it abuts $R$ (and in many cases, $R$ will be a linear feature)

- $A$ is anchored outside $R$-we know for sure that $A$ and $R$ are disjoint.

These anchoring relations are not jointly exhaustive (but are pairwise disjoint), in contrast to the topological relationship models discussed in Section 3.1 above. ${ }^{10}$ So while the anchoring relations are closely related to topology models, the precision of the anchoring relations is in the information space of the discourse model, not in the geometric space of precise locations in the world. Because of this quality, anchoring relations seem attractive for formalizing the comparative proximity meaning of at, which cannot adhere to strict geometric precisifications (Lindkvist 1950). We propose therefore that adding the formal model of Anchoring to our analysis of the meaning of at using a space partitioning with Voronoi cells and the concept of a contrast set will allow us to provide a complete account of the spatial senses for this preposition.

10 Not jointly exhaustive means that the union of all anchoring relations will not equal or exhaust all the possible configurations between two regions. For example, in contrast to topology, when $A$ is anchored in $R$, it can be completely within $R$, touching its border from the inside, or be equal to $R$. In topological terms these are three different relations (inside, coveredBy, and equals; see Figure 1), with different relations between their interior, boundaries and exterior. 


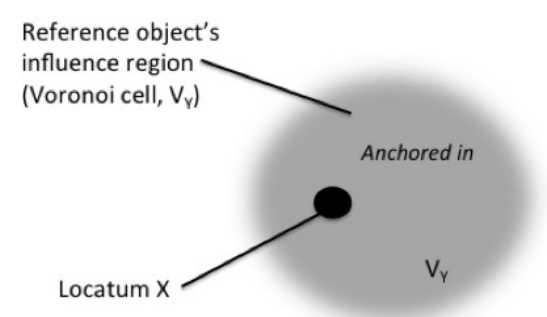

Figure 5 Interpretation of at as anchored in $V_{Y}$, where $V_{Y}$ is the Voronoi cell or influence region of the reference object $Y$. Notice that the represented placement of the locatum is not intended to be exact, but indicates it is confined somewhere in $V_{Y}$, nor do we specify the location of $Y$ here.

Having observed this range of anchoring relations, we argue that at can be formalized with the relation anchoring in as follows:

\section{Definition 2 A locatum $X$ is at a reference object $Y$ if and only if $X$ is anchored in} $V_{Y}$.

In this Definition 2, $V_{Y}$ is the Voronoi cell that corresponds to $Y$ and is part of the Voronoi Diagram created over features of the contrast set $Y$ belongs to - this contrast set being contextually determined. The influence regions - the Voronoi cells - will be possibly different each time at is used, depending on the contrast set of the reference object Y. Formalizing at as anchored in $V_{Y}$ captures the comparative proximity meaning of at through the specification that the locatum is located in the reference object's influence region (Figure 5). Note that this model preserves the comparative, ternary proximity feature described in Section 3. So, if $X$ is anchored in $Y$, it means that $Y$ is chosen because it is the closest reference object to $X$ from among those in the contrast set.

A straightforward example demonstrating the use of at with an anchored in relationship would be an utterance such as The city hall is at the city centre: without being able to specify precise boundaries of the city centre, we state that the city hall is somewhere inside the centre's influence region, as this is defined by contrasting the city centre with other, similarly prominent parts of the city, such as its coastline area, or its suburban areas. This relation would be equivalent to the topological relation 'inside' in Figure 1 except that one cannot prove this without precise boundaries. Other examples of the use of at interpretable in this way are: I am waiting at the tram stop, I will meet you at the library, or the Classics Museum is at the northern end of the city. In all these examples, and in the others we have discussed so far, we see that if trying to place the locatum, it suffices to indicate the Voronoi cell around 
the reference object, from the Voronoi Diagram of the same contrast set. We can then be confident that the locatum is somewhere in that cell; otherwise the description would be made using another reference object.

The definition given above includes the possibility of the locatum being inside the reference object itself (the strict definition of anchored in), or of it covering an area including the interior of the reference object, as in a sloppy description such as The city centre is at the city hall: the city centre is in the influence region of the city hall, but of course includes this building within it.

The shape of the reference object may vary. In an example of a highway which runs alongside the boundaries of a national park (and this can be 'disjoint', 'meet' or even negligibly 'overlap' in any precise location of both features) such as in The Grampians are at the Glenelg Highway, the reference object is elongated in shape, and we might be tempted to suggest that the anchored-alongside relation is a better representation of the spatial relationship referred to here. However, this case too can be accounted for as an instance of anchoring in - it just so happens that the shape of the reference object (the highway) is long and thin, but it is still possible to conceive of the Grampians (locatum $X$ ) as being within the influence region of this reference object.

We would argue, however, that at is rarely or never used to anchor something strictly 'outside' of a reference feature (anchored outside), since there are more appropriate prepositional expressions, such as near, next to or close by, to indicate the strict disjointness of the locations. Anchored outside is explicitly excluded from Definition 2.

This approach combines a well-defined partition of space (Voronoi Diagrams) with a formal model of spatial relations that is actually not precise in geometric space. What is achieved are properly refined acceptable placements of a locatum when it is positioned 'at the reference object' - within the contextually defined Voronoi cell — while remaining true to the often non-exact nature of the spatial configuration described using the preposition at. Conveniently, even when the placement of the locatum is meant to be precise, as in The arrow hit the target at the centre, the inclusive nature of the Voronoi cell accommodates these cases equally well.

Formalizing at this way provides a means of locating features without the need to provide a more specific location. However, by limiting the possible locations to the influence region - here the Voronoi cell — we remain true to the way in which $a t$ is used in natural language place descriptions.

Thinking of at in terms of anchored in $V_{Y}$ has the benefit of overcoming the lack of discriminatory power in topology, orientation and distance illustrated previously. Anchored in, the use of contrast sets, and the Voronoi Diagrams built on them provide a means of formalizing with existing tools a spatial relation that has otherwise eluded 
the existing formalizations, even though it is the most commonly used when referring to a relative location.

Using Anchoring theory to formalize at also helps to clarify the subtle differences between this preposition and some of the other prepositions most closely related to it in meaning, namely in, on and near. For on, the concept of a supporting surface of the reference object is also important, and so while there are cases where on would also be interpreted with anchoring relations, the element of its meaning encoding the force dynamics of gravity (and extended senses based on this) ${ }^{11}$ deserves closer investigation, outside the scope of this work. Using the anchoring relations to define the spatial meaning of in and near however, one would have to consider the actual spatial dimensions of the reference object. Then, definitions for in and near could be given as in Definition 3 and Figure 6. Thus, in Figure 6b the Voronoi cell for interpreting near has a hole, if (or where) the reference object is considered spatially extended, thus capturing a major distinction between near and at, which is that near is not applicable when the location is known to be inside the reference object. It appears that near is more concerned with (outer) distance, while at is more concerned with contact or possible interaction. In contrast, in Figure 6a, the interpretation for in requires precisely that the locatum is situated in the reference object.

Definition 3 The spatial meaning of in and near:

- $X$ is in $Y$, if and only if $X$ is anchored in $E_{Y}$ (Figure 6a); and

- $X$ is near $Y$, if and only if $X$ is anchored in $\left[V_{Y}-E_{Y}\right]$ (Figure 6b).

where $V_{Y}$ is, as before, the Voronoi cell around $Y$ (its influence region) and $E_{Y}$ is the reference object's eigenplace, or the region occupied by the reference object itself in the geometric space.

It becomes clear then, that using at to refer to a location in a context where the focus is on the fact that it is either clearly within or clearly outside and at some (small but definite) distance from the actual (physical boundaries) of the reference object would be misleading in the conversation context, as at is the only one of these prepositions that can actually cover in meaning the whole influence region, including the extent of the reference object itself. Thus for pragmatic reasons, it would be uncooperative to choose at when these more specific meanings are intended, and prepositions in or near would be chosen instead.

Although our observations here are based on linguistic interpretation and formal models, they are relevant to our understanding of cognitive mechanisms. It appears

11 Clearly on can be used in contexts where the supporting role of the reference object is not based on the force dynamics of gravity, for example the picture on the wall; we take such senses to retain the notion of support but to represent an extension from the more prototypical use. 
The preposition at
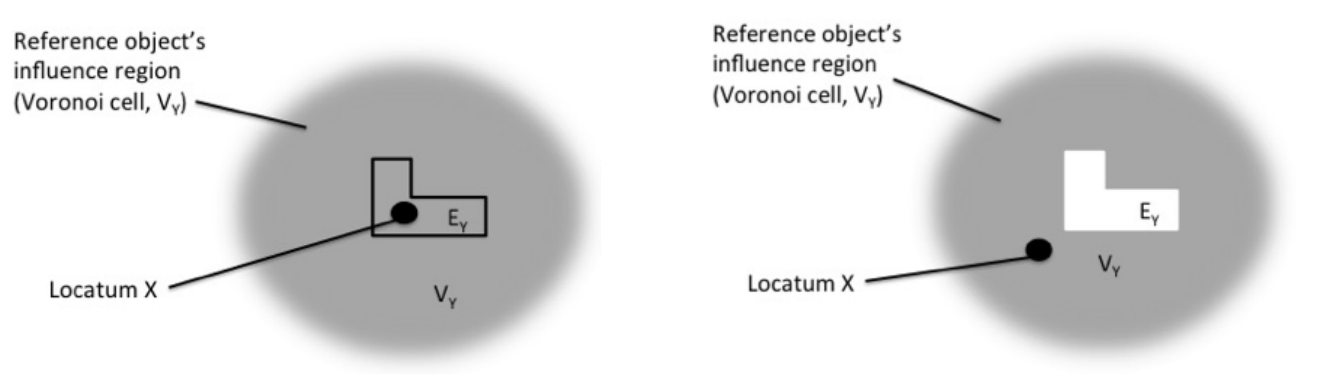

Figure 6 Interpretation of prepositions (a) in, and (b) near using the anchored in relation.

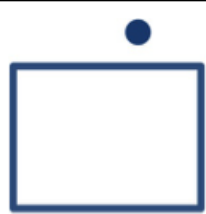

(a)

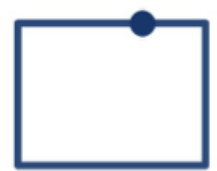

(b)

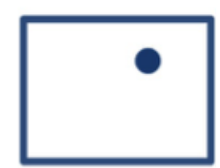

(c)

Figure 7 Distinct topological relations disjoint (a), meet (b) and containedBy (c), but examples can be given where each image is a valid representation of at.

that we could speculatively suggest that the preposition at has a strong spatial connotation (is deeply rooted in the spatial processes in the mind), but one cannot easily depict the relation it implies. While it has a capacity to anchor objects or events with respect to a general location, neither the spatial dimension of the reference object nor the actual spatial relation can be easily depicted graphically. Lindstromberg (1998) links this characteristic to the frequent necessity to mentally 'zoom out' when using at in its spatial sense, so that the relation between locatum and reference object is conceptualised in an abstract way that renders the spatial meaning of at barely discernable: it could be near the landmark but not touching it, near and touching it, or simply co-located with (exactly, partially or surrounded by) the landmark (Figure 7). Thus, image schemata (Johnson 1987) as they are taken to underlie, for example, the topological relations that represent the meaning of in (container schema) or on (surface schema), cannot be readily or nonabstractly used to represent the meaning of at. Hence, we argue that at conveys a spatial meaning, but not an easy to visualize image schema. In visual terms, at remains ambivalent. This is perhaps why it has been difficult to capture the meaning of at within cognitive semantics. A cognitive grounding for the preposition must point to linguistic-cognitive schematizations (Talmy 1983) that are not easily imaginable. 
Vasardani, Stirling and Winter

5 Comparing this account to Zwarts \& Y. Winter 2000

This paper started out from the hypothesis that the meaning of at can be formally captured by tools of GIScience. It considered known formal frameworks for capturing the meaning of qualitative spatial relations: topological, orientation and distance models as they have been suggested for precise locations, and Anchoring as it has been suggested for locations that are not precisely known. In each of these categories we studied the discriminatory power of at to identify cases where at may have informational content.

We have demonstrated that the primary meaning of $a t$ is a comparative distance relationship, best captured by a novel formal model incorporating contrast sets, partitioning space using Voronoi cells, and the anchored in relation. In combination with directed activity predicates at is interpreted in the same way along a vector from source to goal. The comparative distance meaning is related to a contrast set of features relevant in a current communication context. As the focus moves, the relevant contrast set can switch. Having established that influence regions, rather than quantitative distances or qualitative nearness, best model the comparative proximity character of at, we introduced the notion of anchored in $V_{Y}$. By focusing on reference objects that belong to the same contrast set, we argue that their influence regions can then be represented by Voronoi Diagrams that are constructed from the locations of the objects belonging to the same contrast set. We therefore argue that pairing contrast sets and anchoring seems to be the most effective way of formalizing the meaning of at using current tools available from GIScience as it allows for the locatum's location to remain underspecified, while at the same time enabling reasoning with existing tools.

We have therefore proposed a formal model within the terms of GIScience underpinning the semantics of $a$. The model allows us to capture the intuitions that have led to previous linguistic semantic accounts describing the spatial meaning of at as specifying a relation of contiguity or proximity between a locatum and a location conceived of as a point. We have confirmed that it is with respect to distance rather than topology that at has discriminatory power. The intuition of a point-like location remains in our use of a Voronoi Diagram of seeds to model the notion of a prototype or centre characterising a place with a region of influence determined by a contextually-specified contrast set. Our more detailed representation of an anchoring relationship between the locatum and location, defined over Voronoi cells, allows us to capture the constraints on at such that it is not used for relationships of strict outside-ness, but is sufficiently underspecified to account for the wide range of actual spatial configurations which can be described using this preposition.

The formal mechanisms we have been discussing concern the proper ontological structure needed to define the meaning of the preposition at and, we suggest, poten- 
tially of other spatial prepositions also (we sketched the basis for an account for near and in in Section 4; see also below). We have indicated that there is evidence from the GIScience literature that these models are on the right track in terms of their correspondence with people's cognitive representations of the qualitative spatial relations relevant to these prepositions.

Such an account could be embedded as a formal lexical semantics for the preposition at within a model-theoretic semantics for natural language, such as, for example, that assumed by Zwarts \& Y. Winter (2000). Instead of the sets of vectors and vector spaces they propose, we argue for the addition to the ontology of a domain of Voronoi spaces (as well as assuming a domain of points as they do) and operations of anchoring which apply to these. The preposition at can then be taken to denote a function that applies to the reference object's eigenplace and returns its Voronoi cell. In addition we need to assume that a contrast set for the Voronoi cell is determined pragmatically within the context of the discourse in which the preposition occurs.

More formally, Zwarts \& Y. Winter (2000) provide the definition below for the prepositions on, at and near. In their analysis, these prepositions give rise to locations strictly outside the reference object's eigenplace; hence, if $v$ is a vector used to present the relation between locatum and reference object, the eigenplace of which is $A$, they use a relation $\operatorname{ext}(v, A)$ in their definitions of the relations to imply that the locatum remains external to $A$. The only other restriction on the meaning of the prepositions is a specification of a condition on the length of the vector $v$ which describes the distance between the reference object and the locatum. In their model, prepositions on and at are given the same definition - are indistinguishable — as they note "We interpret on and at as requiring almost zero distance between the objects. [...] The differences between on and at [...] and many other interesting meaning aspects are again ignored" (p. 180).

Definition 4 The prepositions on, at and near according to Zwarts \& Y. Winter (2000):

- on, at: on' $=\boldsymbol{a t}, \stackrel{\text { def }}{=} \lambda A \cdot \lambda v \cdot \operatorname{ext}(v, A) \wedge|v|<r_{0}$

- near: near $\stackrel{\text { def }}{=} \lambda A \cdot \lambda v \cdot \operatorname{ext}(v, A) \wedge|v|<r_{1}$

where $r_{0}$ and $r_{1}$ are two small positive numbers $r_{0} \approx 0$ and $r_{0} \ll r_{1}$.

We now sketch a method for formalizing at within compositional semantics using Anchoring theory. This serves as a starting point and allows us to make comparisons with Zwarts \& Y. Winter's (2000) formalization, such as enabling a differentiation between at and $o n$. 
Let $S_{c}=\{1 \ldots n\}$ be the given contrast set with $n$ elements. Then $V_{i}$, with $i \in S_{c}$ is the Voronoi cell that corresponds to seed $i$, and $\bigcup_{i}^{n} V_{i}$ is the Voronoi Diagram of $S_{c}$. Then the $V_{i}$ are the partitions of precise (or coordinized) geographic space. Since we are using Anchoring Theory to formalize at, what we know about objects that are located (locata) is information about their relative locations and spatial relations with other objects (relata), rather than their exact locations. In fact, when using at with anchoring relations, instead of defining a location, we are actually defining a set of locations. For any object $x$ then, $\operatorname{loc}(x)$ denotes the set of all possible locations where $x$ can be found.

In anchoring theory, any relation is formalized as an ordered pair $(t, R)$, where $t$ is any of the anchoring relations defined in Definition 1 and here abbreviated to just the differing name part, respectively, so $t \in\{\mathrm{in}$, over, alongside, outside $\}$ and $R$ is a region in precise geographic space. Then, for the purpose of formalizing $a t, t=\mathrm{in}$ and $R=V_{Y}$ such that:

$$
x \text { at } Y \leftrightarrow\left(\text { in }, V_{Y}\right) \in \operatorname{loc}(x)
$$

where $V_{Y}$ is the Voronoi cell with seed $Y$, and $Y$ is an element of the given contrast set $S_{c}$. In case a quantifier such as exactly is used in conjunction with at, then $R$ is the eigenplace of $Y, E_{Y}$, such that:

$$
x \text { exactly_at } Y \leftrightarrow\left(\text { in }, E_{Y}\right) \in \operatorname{loc}(x)
$$

Furthermore, in line with the account sketched in Section 4, we can define in and near as follows:

$$
\begin{gathered}
x \text { in } Y \leftrightarrow\left(\text { in }, E_{Y}\right) \in \operatorname{loc}(x) \\
x \text { near } Y \leftrightarrow\left(\text { in, }, V_{Y}-E_{Y}\right) \in \operatorname{loc}(x)
\end{gathered}
$$

Returning the focus to $a t$, we can start introducing some rules to our theory, such as:

Rule 1 If $x$ at $Y$, and $x$ at $Z$, then $V_{Y}$ and $V_{Z}$ have all or some parts in common, which means that $Y$ and $Z$ belong to different contrast sets - since the Voronoi cells of the same partition are pairwise disjoint:

$$
\left(\text { in }, V_{Y}\right) \in \operatorname{loc}(x) \wedge\left(\text { in }, V_{Z}\right) \in \operatorname{loc}(x) \rightarrow V_{Y} \cap V_{Z} \neq \varnothing
$$

Rule 2 If $x$ at $Y$, and $Y$ is part of $Z$, then $x$ is at $Z$ as well:

$$
\left(\text { in }, V_{Y}\right) \in \operatorname{loc}(x) \wedge(Y \subseteq Z) \rightarrow\left(\text { in }, V_{Z}\right) \in \operatorname{loc}(x)
$$

It is clear then that our formalization differentiates between $a t$, on and near in a way that the formalization presented by Zwarts \& Y. Winter (2000) does not. Their theory's dependence on vector lengths imposes limitations on how defining at can 
differ from defining on, while introducing the ext() relation and the fact that they require the vector for these two prepositions to be almost, but not quite zero, implies that the exact co-location with the reference object's eigenplace is excluded from $a t$ 's or on's definitions. However, at can be used for objects co-located with the reference object as well as close to the reference object, in other words, the entire set of locations covered by the influence region or Voronoi cell of the reference object. This is clearly indicated by the definition provided using the anchoring in relation in combination with the Voronoi cells. It is in contrast then to Zwarts \& Y. Winter's account, in that it does not require that at be outward oriented (see Definition 4): at appears to be valid both outwards (near) and inwards (in, on). On the other hand, the preposition on implies a supporting surface, something that is not specified in Zwarts \& Y. Winter's theory, which as we have seen provides the same definition for both at and on. While we have not formally defined on, it is clear in our definition that at can potentially position the locatum in the $V_{Y}-E_{Y}$ area, for which on cannot be used. Finally, on our definition of near, the locatum can be somewhere in the $V_{Y}-E_{Y}$ area, but not in $E_{Y}$. This definition is cleaner, not requiring pre-knowledge of the relation between the lengths of different vectors for differentiating between near and at.

The formal models presented here are intended to represent the core lexical semantic meaning for at and we assume that they will be augmented with a NeoGricean pragmatic account which will help specify the way in which speakers make choices between at and more informationally specific prepositions in particular contexts of use. The models applied here in the investigation of the semantics of at may have value for the formal interpretation of other locative prepositions as well. It remains for future work to explore the broader applicability and generalizations of the presented formal model in more detail.

\section{Specific cases and over-generation}

We have proposed an account that captures the broad and vague uses of the preposition at; however, as we have acknowledged, at is not always a possible, let alone appropriate, choice for specifying the location of a given locatum. In this section we provide a detailed consideration of some types of situation where this is the case.

We have previously noted that at can be used in place of more specific prepositions, such as in or on. However, there are cases where at is not used in place of these prepositions. For example, at is a dispreferred alternative to in in certain cases where the location of the locatum is completely inside the two- or three-dimensional surface enclosed within the reference object's physical boundaries (or its eigenplace in Gärdenfors's words). Take for instance the following sentences: I left my books at 
your house versus I left my books in your study, where the converse possibilities are less acceptable: ?I left my books in your house; ? left my books at your study.

The first example, assuming the books are within the eigenplace identified with the house, is predicted by our account which allows for at to be used if the locatum is in the relation of anchored in the region $E_{Y}$ - the eigenplace of $Y$, where $Y$ is the house. The use of in is also predicted here by the formal definition given in our model. The interesting question is then why the preference for this particular sentence is for at to be used over in. Arguably, in is not ruled out here in terms of overall acceptability — clearly we can say something like My books are somewhere in your house. The issue is one of construal of the situation and whether the nature of the locational relation between the locatum and a reference object conceived of as a 3-dimensional entity is relevant in context - is it important where exactly the books are inside the house?

In the second example, it is clearly easier to construe the study as a 3-dimensional spatial entity within which it is relevant to consider the precise location of the books. Intuitively it appears that use of at in such a context will be possible only if your study refers to a maximal space associated with you, for instance, if you have a study within a University college, where the study can be viewed as a self-contained landmark, the dimensionality of which is not relevant.

Similar observations can be made for examples with the preposition on. Where it is known and relevant that the locatum is physically supported by the reference object, there will be a preference for on over at. Take for example the following sentences: Sam is at his desk versus Sam was on his desk when I found him. While the first case refers to the functional coincidence of the locatum with the reference object, the second case indicates that physically the locatum was supported by the reference object, therefore, the spatial preposition on is used. Similarly, Sam is at the sofa highlights a construal of the sofa as a mere landmark with reference to which Sam can be placed while, in comparison, Sam is on the sofa portrays a more complex and more precisely specified locational relationship for which a topological representation can be defined.

Both cases relate to the general consensus about the core spatial meaning of at, that it indicates a proximity or co-location of two entities that are imagined as points (rather than 2- or 3-dimensional entities), or where the dimensionality is simply not an issue, and where the precise nature of the spatial relationship between the locatum and the reference object is not known or not relevant. We have modelled this property of at in comparison to the other prepositions considered, by arguing that unlike these prepositions, at places entities at a non-topologically-defined location, and have argued that this is why it is difficult to assign at to only one of the distinct formal topological relations as defined in GIScience (similarly, for direction or distance relations). The location is defined in terms of Voronoi cells, the extent of which is 
determined in a relative manner by their relationship with other cells of members of a relevant contrast set. Since space then is not topologically defined, we should not be surprised that the actual dimensionality of the locatum and of the reference object is not relevant. Where the dimensionality is relevant, by neo-Gricean principles such as the Q(uantity)- and I(nformativeness)-principles (in effect, say neither more nor less than is required), the speaker is obligated to use an informationally richer preposition for which the semantics makes reference to the topological (or even more generally, geometrical) properties of the related entities (Huang 2015, Levinson 2000). To sketch the basis of such an account without fully developing it, we can suggest that at is undetermined for topological information, and that at and the topologically more precise prepositions on and in participate in a locational Horn-scale or Qscale $<\{$ in, on $\}$, at $>$ such that use of the less specific preposition implicates that the precise topological relationship is either unknown or inapplicable, or perhaps, that the speaker's primary intention is to characterise the location of some entity via implicit contrast to some other location(s). A similar argument can be made for a choice of near over at as distance relation; the former will be used when it is necessary to indicate that the locatum is not co-located with the reference object, but rather at a small distance from it.

While we suggest that the model presented here allows the interpretation of any given (valid) spatial use of $a t$, it possibly overgenerates the production of sentences using at. For example, the case of I am at 15 Elm Street is a valid use (and to be interpreted with respect to a contextually determined contrast set, for instance of other relevant addresses). However, native speaker intuitions suggest that I am at Australia is not generally considered a valid use, but it will still be accepted by our model, assuming the pragmatic mechanism for generating a relevant contrast set can provide one (e.g., a set of other relevant countries). Our account does, however, correctly imply that if such a usage could be contextualised, it would be in a context where we can 'zoom out' to view Australia as one point in a contrast set of comparable points, for instance as one of a sequence of destinations on a route. A representation of Australia on a board game would be an appropriate context, or perhaps an utterance by recently returned astronaut Scott Kelly made while orbiting the Earth. The appropriate modelling of these restrictions will be a matter for pragmatics. ${ }^{12}$

12 Consistent with these observations, Herskovits (1986) suggests that such examples are ruled out by a constraint to the effect that large reference objects are harder to conceptualise as locations, which would reflect the relative paucity of contexts in which they can be viewed as zero-dimensional. 
Vasardani, Stirling and Winter

\section{Conclusions}

At has generally been considered to express a spatial locational relation of such generality that it is sometimes thought of as simply the location relation, akin to the implicit relation of location found in a locative expression without an associated preposition (Where are you? - Myers ${ }^{13}$ ). This relation has been classified within both linguistic semantics and GIScience as topological and as involving essential components of coincidence or co-location and zero-dimensionality of the co-located entities, among a number of other constraints that various authors have proposed.

Yet it is well understood that, strictly interpreted, coincidence is both too restrictive and too underspecified to account for many uses of at. Herskovits (1986), for instance, accounts for very frequently occurring, 'almost true' uses such as Mary is at the gate (see also Table 1b) by combining an 'ideal' abstract geometric meaning with processes allowing for 'relational tolerance' or permitted deviations from this - to give rise to a more comprehensive informal gloss for at to the effect that the locatum is specified to be 'very close to but with a certain tolerance' or 'in the closest possible relationship with' the reference object (cf. the 'almost zero' vector in the account of Zwarts \& Y. Winter (2000)). The model proposed here provides a formal mechanism for capturing the intuitions expressed in these attempts to account for the degree of tolerance allowed in the locational relationship between the locatum and the reference object by $a t$.

Thus, what we have suggested here is that the formal models in use in GIScience offer tools which allow us to more accurately model the range of uses of this preposition (and possibly others), removing the need to capture a vast class of uses as departures from the norm, or to rely on vague notions such as an 'almost zero' distance.

At the same time, a detailed consideration of the range of uses available for at from a more linguistic perspective has allowed us to improve upon previous GIScience accounts. Contrary to prior assumptions, we have systematically detailed how at lacks topological discrimination. Our account embraces aspects of the inherent vagueness of the meaning of at - the underspecified nature of the real world configuration between locatum and reference object, and the range of possible 'distances' allowable between these two entities. Our assumption is that the contrast sets assumed to be a critical aspect of the account (and the relative size and granularity of the choices in the contrast sets) are contextually determined and a matter of perspective or framing. Also, pragmatically, at is used when the focus of attention is not on encoding more specific spatial relations between locatum and reference object but rather on the specification of the reference object as a relevant location per se (from among a set of possibly relevant locations). It is for this reason that

13 Myers is a department store in Australia. 
The preposition $a t$

at appears to offer degrees of 'tolerance' greater than, for example, the preposition in, which highlights the nature of the relation. We have also sketched how relevant meanings of these alternative prepositions can be represented in our model.

We have thus provided a formal account for what it means to be 'contiguous or proximal' with a reference object, which underpins the pragmatic factors determining the exact nature of the distance relationship between locatum and reference object, and which moves ahead our understanding of the meaning of at and of spatial prepositions more generally, leaving some additional nuances to be considered in further work.

This contribution clarifies the meaning of at. It links at to formal models of qualitative spatial relations, highlighting its particularities, and in this way enabling improved automatic interpretations of this preposition.

\section{Acknowledgments}

This research has been supported by the Australian Research Council (LP120200130).

\section{References}

Bennett, David C. 1975. Spatial and temporal uses of English prepositions: An essay in stratificational semantics (Longman Linguistic Library 17). London: Longman. 235 pp. http://dx.doi.org/10.1016/0024-3841(76)90051-6.

Bloom, Paul \& Frank Keil. 2001. Thinking through language. Mind \& Language 16(4). 351-367. http://dx.doi.org/10.1111/1468-0017.00175.

Brennan, Jane \& Eric Martin. 2002. Foundations of a formalism of nearness. In Bob McKay \& John Slaney (eds.), Advances in artificial intelligence, vol. 2557 (Lecture Notes in Artificial Intelligence), 71-82. Berlin: Springer. http://dx.doi. org/10.1007/3-540-36187-1_7.

Bryant, David J. 1997. Representing space in language and perception. Mind \& Language 12(3). 239-264. http://dx.doi.org/10.1111/j.1468-0017.1997.tb00073. $\mathrm{x}$.

Burrough, Peter A. \& Andrew Frank. 1996. Geographic objects with indeterminate boundaries. CRC Press. 374 pp. http://dx.doi.org/10.1111/j.1467-9671.1997. tb00064.x.

Carlson-Radvansky, Laura A. \& Darrell Irwin. 1994. Reference frame activation during spatial term assignment. Journal of Memory and Language 33(5). 646671. http://dx.doi.org/10.1006/jmla.1994.1031.

Cienki, Alan J. 1989. Spatial cognition and the semantics of prepositions in English, Polish, and Russian. Vol. 237 (Slavistische Beiträge 237). Munich: Verlag Otto Sagner. 172 pp. 
Vasardani, Stirling and Winter

Clark, Herbert H. \& Deanna Wilkes-Gibbs. 1986. Referring as a collaborative process. Cognition 22(1). 1-39. http://dx.doi.org/10.1016/0010-0277(86)900107.

Cohn, Anthony G. \& Jochen Renz. 2008. Qualitative spatial representation and reasoning. In Frank van Harmelen, Vladimir Lifschitz \& Bruce Porter (eds.), Handbook of knowledge representation, 551-596. Elsevier. http://dx.doi.org/10. 1016/S1574-6526(07)03013-1.

Cresswell, Tim. 2004. Place: A short introduction. Malden, MA: Wiley-Blackwell. $168 \mathrm{pp}$.

Cui, Zhan, Anthony G. Cohn \& David A. Randell. 1993. Qualitative and topological relationships in spatial databases. In David Abel \& Beng Chin Ooi (eds.), Advances in spatial databases, vol. 692 (Lecture Notes in Computer Science), 296-315. Springer. http://dx.doi.org/10.1007/3-540-56869-7_17.

Cuyckens, Hubert. 1984. At: A typically English preposition. Poznan Studies in Contemporary Linguistics 19. 49-64.

Dube, Matthew \& Max J. Egenhofer. 2012. An ordering of convex topological relations. In Ningchuan Xiao, Mei-Po Kwan, Michael F. Goodchild \& Shashi Shekhar (eds.), Geographic information science, vol. 7478 (Lecture Notes in Computer Science), 72-86. Springer Berlin Heidelberg. http://dx.doi.org/10. 1007/978-3-642-33024-7_6.

Egenhofer, Max J. 2015. Qualitative spatial-relation reasoning for design. In John S. Gero (ed.), Studying visual and spatial reasoning for design creativity, 153-175. Springer Netherlands. http://dx.doi.org/10.1007/978-94-017-9297-4_9.

Egenhofer, Max J., Andrew U. Frank \& Jeffrey P. Jackson. 1989. A topological data model for spatial databases. In Alejandro Buchmann, Oliver Günther, Terence R. Smith \& Yuan-Fang Wang (eds.), Design and implementation of large spatial databases, vol. 409 (Lecture Notes in Computer Science), 271-286. New York: Springer.

Egenhofer, Max J. \& Robert D. Franzosa. 1991. Point-set topological spatial relations. International Journal of Geographical Information Systems 5(2). 161-174. http://dx.doi.org/10.1080/02693799108927841.

Egenhofer, Max J. \& David M. Mark. 1995. Naive geography. In Andrew U. Frank \& Werner Kuhn (eds.), Spatial information theory, vol. 988 (Lecture Notes in Computer Science), 1-15. Berlin: Springer. http://dx.doi.org/10.1007/3-54060392-1_1.

Egenhofer, Max J., Jayant Sharma \& David M. Mark. 1993. A critical comparison of the 4-intersection and 9-intersection models for spatial relations: Formal analysis. In Robert B. McMaster \& Marc P. Armstrong (eds.), Auto-Carto 11, vol. 11, 1-11. ASPRS. 
The preposition $a t$

Evans, Vyvyan. 2010. From the spatial to the non-spatial: The state lexical concept of in, on and at. In Vyvyan Evans \& Paul Chilton (eds.), Language, cognition and space: The state of the art and new directions. London: Equinox. http: //dx.doi.org/10.1558/equinox.22031.

Frank, Andrew U. 1991. Qualitative spatial reasoning about cardinal directions. In David M. Mark \& Denis White (eds.), Auto-Carto 10 (ACSM-ASPRS), 148-167. http://dx.doi.org/10.1007/978-3-642-46752-3_17.

Frank, Andrew U. 1996. Qualitative spatial reasoning: Cardinal directions as an example. International Journal of Geographical Information Systems 10(3). 269-290. http://dx.doi.org/10.1080/02693799608902079.

Frank, Andrew U. \& David M. Mark. 1991. Language issues for geographical information systems. In David J. Maguire, Michael Goodchild \& David Rhind (eds.), Geographical information systems: Principles and applications, vol. 1, 147-163. London: Longman.

Freksa, Christian. 1991. Qualitative spatial reasoning. In David M. Mark \& Andrew U. Frank (eds.), Cognitive and linguistic aspects of geographic space (NATO ASI Series D: Behavioural and Social Sciences), 361-372. Dordrecht, NL: Kluwer Academic Press. http://dx.doi.org/10.1007/978-94-011-2606-9_20.

Freksa, Christian. 1992. Using orientation information for qualitative spatial reasoning. In Andrew U. Frank, I. Campari \& U. Formentini (eds.), Theories and models of spatio-temporal reasoning in geographic space, vol. 639 (Lecture Notes in Computer Science 639), 162-178. Berlin: Springer. http://dx.doi.org/10.1007/3540-55966-3_10.

Galton, Antony \& James Hood. 2005. Anchoring: A new approach to handling indeterminate location in GIS. In Anthony G. Cohn \& David M. Mark (eds.), Spatial information theory, vol. 3693 (Lecture Notes in Computer Science), 1-13. Berlin: Springer. http://dx.doi.org/10.1007/11556114_1.

Gärdenfors, Peter. 2014. The geometry of meaning. Cambridge, MA: The MIT Press. Hall, Mark M., Christopher B. Jones \& Philip Smart. 2015. Spatial natural language generation for location description in photo captions. In Sara Irina Fabrikant, Martin Raubal, Michela Bertolotto, Clare Davies, Scott Freundschuh \& Scott Bell (eds.), Spatial information theory, vol. 9368 (Lecture Notes in Computer Science), 196-223. Springer International Publishing. http://dx.doi.org/10.1007/ 978-3-319-23374-1.

Hausdorff, Felix. 1914. Grundzüge der Mengenlehre. Leipzig: Veit \& Company.

Hernández, Daniel, Eliseo Clementini \& Paolino Di Felice. 1995. Qualitative distances. In Andrew U. Frank \& Werner Kuhn (eds.), Spatial information theory, vol. 988 (Lecture Notes in Computer Science), 45-57. Berlin: Springer. http: //dx.doi.org/10.1007/3-540-60392-1_4. 
Vasardani, Stirling and Winter

Herskovits, Annette. 1985. Semantics and pragmatics of locative expressions. Cognitive Science 9(3). 341-378. http://dx.doi.org/10.1207/s15516709cog0903_3.

Herskovits, Annette. 1986. Language and spatial cognition. Cambridge, UK: Cambridge University Press.

Huang, Yan. 2015. Neo-Gricean pragmatic theory of conversational implicature. In Bernd Heine \& Heiko Narrog (eds.), The Oxford handbook of linguistic analysis, vol. 1 (Oxford Handbooks in Linguistics), 615-639. Oxford University Press. http://dx.doi.org/10.1093/oxfordhb/9780199544004.013.0024.

Jackendoff, Ray. 1983. Semantics and cognition (Current studies in linguistics series). Cambridge, MA: MIT Press.

Johnson, Mark. 1987. The body in the mind: The bodily basis of meaning, imagination, and reason. Chicago, IL: The University of Chicago Press.

Knas, Iwona. 2006. Polish equivalents of spatial at. In Third ACL-SIGSEM workshop on prepositions, 9-16. Association for Computational Linguistics. http://dx.doi. org/10.3115/1621431.1621433.

Knauff, Markus, Reinhold Rauh \& Jochen Renz. 1997. A cognitive assessment of topological spatial relations: Results from an empirical investigation. In Stephen C. Hirtle \& Andrew U. Frank (eds.), Spatial information theory, vol. 1329 (Lecture Notes in Computer Science), 193-206. Springer. http://dx.doi.org/10. 1007/3-540-63623-4_51.

Kuipers, Benjamin J. 1977. Representing knowledge of large-scale space. Cambridge, MA: PhD. 126 pp.

Kuipers, Benjamin J. 1994. Qualitative reasoning: Modeling and simulation with incomplete knowledge. Cambridge, MA: The MIT Press. http://dx.doi.org/10. 1016/0005-1098(89)90099-X.

Landau, Barbara \& Ray Jackendoff. 1993. What and Where in spatial language and spatial cognition. Behavioral and Brain Sciences 16(2). 217-238. http: //dx.doi.org/10.1017/S0140525X00029733.

Leech, Geoffrey N. 1970. Towards a semantic description of English. Bloomington, UK: Indiana University Press. 304 pp.

Levinson, Stephen C. 1996. Frames of reference and Molyneux's question: Crosslinguistic evidence. In Paul Bloom, Mary A. Peterson, Lynn Nadel \& Merrill F. Garrett (eds.), Language and space, 109-169. Cambridge, MA: The MIT Press.

Levinson, Stephen C. 2000. Presumptive meanings: The theory of generalized conversational implicature. Cambridge, MA: MIT Press. 516 pp.

Levinson, Stephen C. 2003. Space in language and cognition. Cambridge, UK: Cambridge University Press. 389 pp.

Lindkvist, Karl-Gunnar. 1950. Studies on the local sense of the prepositions in, at, on, and to, in modern English. Lund, Sweden: C. W. K. Gleerup. 
The preposition $a t$

Lindstromberg, Seth. 1998. English prepositions explained. John Benjamins Publishing Company. 328 pp.

Mador-Haim, Sela \& Yoad Winter. 2015. Far from obvious: The semantics of locative indefinites. Linguistics and Philosophy 38(5). 437-476. http://dx.doi.org/10. 1007/s10988-015-9175-y.

Mark, David M. \& Max J. Egenhofer. 1994. Calibrating the meanings of spatial predicates from natural language: Line-region relations. In Thomas C. Waugh \& Richard G. Healey (eds.), Advances in GIS research, 538-553.

Mark, David M. \& Andrew U. Frank. 1991. Language, cognitive science, and geographic information systems. Technical Report 90-10, National Center of Geographic Information Analysis.

Mark, David, David Comas, Max J. Egenhofer, Scott M. Freundschuh, Michael Gould \& Joan Nunes. 1995. Evaluating and refining computational models of spatial relations through cross-linguistic human-subjects testing. In Andrew Frank \& Werner Kuhn (eds.), Spatial information theory - a theoretical basis for GIS, vol. 988 (Lecture Notes in Computer Science), 553-568. Berlin: Springer. http://dx.doi.org/10.1007/3-540-60392-1_36.

Mark, David \& Max Egenhofer. 1995. Topology of prototypical spatial relations between lines and regions in English and Spanish. In Auto-Carto 12, 245-254. Charlotte, NC.

Mark, David, Andrew U. Frank, Max J. Egenhofer, Scott M. Freundschuh, Matthew McGranaghan \& R. Michael White. 1989. Languages of spatial relations. Santa Barbara, CA: National Center of Geographic Information Analysis.

Munnich, Edward, Barbara Landau \& Barbara Anne Dosher. 2001. Spatial language and spatial representation: A cross-linguistic comparison. Cognition 81(3). 171208. http://dx.doi.org/10.1016/S0010-0277(01)00127-5.

Piñón, Christopher. 1993. Paths and their names. In Katherine Beals, Gina Cooke, David Kathman, Kita Sotaro, Karl-Erik McCullough \& David Testen (eds.), Papers from the 29th regional meeting of the Chicago linguistic society, vol. 2, 287-303. Chicago, IL: CLS.

Quirk, Randolph, Sidney Greenbaum, Geoffrey N. Leech \& Jan Svartvik. 1985. A comprehensive grammar of the English language. Longman. $1800 \mathrm{pp}$.

Randell, David A., Zhan Cui \& Anthony G. Cohn. 1992. A spatial logic based on regions and connection. In Ronald Brachmann, Hector Levesque \& Ray Reiter (eds.), Third international conference on the principles of knowledge representation and reasoning, 165-176. Morgan Kaufmann.

Renz, Jochen, Reinhold Rauh \& Markus Knauff. 2000. Towards cognitive adequacy of topological spatial relations. In Spatial cognition II, vol. 1849 (Lecture Notes in Artificial Intelligence), 184-197. Berlin: Springer. http://dx.doi.org/10.1007/3540-45460-8_14. 
Shannon, Claude E. 1948. A mathematical theory of communication. The Bell System Technical Journal 27(7). 379-423 and 623-656. http://dx.doi.org/10.1002/j. 1538-7305.1948.tb01338.x.

Shannon, Claude E. \& Warren Weaver. 1949. The mathematical theory of communication. Chicago: University of Illinois Press.

Snider, Todd. 2010. The semantics of prepositions: An exploration into the uses of at and to. Honors thesis, Department of Philosophy, Carnegie Mellon University.

Talmy, Leonard. 1983. How language structures space. In Herbert Pick \& Linda Acredolo (eds.), Spatial orientation: Theory, research, and application, 225-282. New York: Plenum Press. http://dx.doi.org/10.1007/978-1-4615-9325-6_11.

Tyler, Andrea \& Vyvyan Evans. 2003. The semantics of English prepositions: Spatial scenes, embodied meaning, and cognition. Cambridge University Press. 270 pp. Vasardani, Maria, Stephan Winter, Kai-Florian Richter, Lesley Stirling \& Daniela Richter. 2012. Spatial interpretations of preposition at. In First ACM SIGSPATIAL international workshop on crowdsourced and volunteered geographic information, 46-53. New York: ACM Press. http://dx.doi.org/10.1145/2442952. 2442961.

Voronoi, Georgy. 1908. Nouvelles applications des paramètres continus àa la théorie des formes quadratiques, deuxiéme memoire, recherche sur les parallelloédres primitifs. Journal für die Reine und Angewandte Mathematik 134. 198-287.

Winter, Stephan \& Christian Freksa. 2012. Approaching the notion of place by contrast. Journal of Spatial Information Science 2012(5). 31-50. http://dx.doi. org/10.5311/JOSIS.2012.5.90.

Worboys, Michael F. 2001. Nearness relations in environmental space. International Journal of Geographical Information Science 15(7). 633-652. http://dx.doi.org/ 10.1080/13658810110061162.

Wunderlich, Dieter. 1991. How do prepositional phrases fit into compositional syntax and semantics? Linguistics 29(4). 591-621. http://dx.doi.org/10.1515/ling.1991. 29.4.591.

Zwarts, Joost. 2005. Prepositional aspect and the algebra of paths. Linguistics and Philosophy 28(6). 739-779. http://dx.doi.org/10.1007/s10988-005-2466-y.

Zwarts, Joost \& Yoad Winter. 2000. Vector space semantics: A model-theoretic analysis of locative prepositions. Journal of Logic, Language and Information 9(2). 169-211. http://dx.doi.org/10.1023/A:1008384416604. 
The preposition at

Maria Vasardani

Department of Infrastructure Engineering

Lesley Stirling

The University of Melbourne

Parkville, VIC 3010, Australia

maria.vasardani@unimelb.edu.au

School of Languages and Linguistics

䈝

Stephan Winter

Department of Infrastructure Engineering

The University of Melbourne

Parkville, VIC 3010, Australia

lesleyfs@unimelb.edu.au

The University of Melbourne

Parkville, VIC 3010, Australia

winter@unimelb.edu.au 